\title{
Gerichte gegen Gesetzgeber? - Der Klimawandel in den Gerichtssälen
}

\author{
Manuela Niehaus
}

Abstract: Nachdem ein Gericht in Den Haag die niederländische Regierung 2015 zu mehr Klimaschutz verpflichtete, gibt es keine Zweifel mehr: der Klimawandel ist in den Gerichten angekommen. Durch strategische Prozessführung, insbesondere unter Rückgriff auf die Menschenrechte, setzen Kläger:innen teils erfolgreich mehr Klimaschutz durch. Es stellt sich aber die Frage, ob Gerichte dadurch illegitim neues Klimarecht schaffen und somit gegen den Gewaltenteilungsgrundsatz verstoßen. Der Beitrag zeigt indes, dass Gerichte nie nur Recht anwendeten, sondern auch immer schon erzeugten. Vor dem Hintergrund der inter- und intragenerationellen Dimensionen des globalen Klimawandels wird ibre Stellung im Spannungsfeld zwischen Volkssouveränität und Menschenrechten diskutiert. Es zeigt sich, dass es sich zwar um rechtliches Neuland handelt, aber der Vorwurf des Verstoßes gegen den Gewaltenteilungsgrundsatz entkräftet wird. Vielmehr können Klimaklagen den Zugang zum Recht für diejenigen obne Stimme im politischen Willensprozess öffnen und damit zu einer nachhaltigeren Demokratie beitragen.

Mit dem Abschluss des Paris Agreement 2015 hofften viele Umweltschützer:innen, dass die Regierungen nun endlich den notwendigen Aufschwung bekommen würden, um mutig und entschlossen gegen den Klimawandel vorzugehen. Diese Hoffnungen sind weitestgehend enttäuscht worden: die Emission Gap Reports der vergangenen Jahre legen offen, dass nach wie vor zu viele Treibhausgase (THG) in die Atmosphäre gelangen. ${ }^{1}$

Seit einigen Jahren protestieren deswegen junge Menschen bei den „Fridays for Future“-Demonstrationen für ihre Zukunft, während andere Protestgruppen wie Extinction Rebellion ganze Städte lahmlegen, um die Klimakrise medienwirksam in den Mittelpunkt zu rücken. Neben den Protesten sind es aber vor allem die Gerichte, die mit spektakulären Urteilen

1 Vgl. UNEP, Emissions Gap Report 2020, wonach - trotz eines kurzzeitigen Einbruchs der THG-Emission aufgrund der Corona Pandemie - die Welt weiterhin auf eine Erhöhung auf $3^{\circ} \mathrm{C}$ statt der angestrebten $1.5-2^{\circ} \mathrm{C}$ zusteuert. 
für mehr Klimaschutz gesorgt haben und die damit im Kampf gegen den Klimawandel zu bedeutsamen Akteuren werden. ${ }^{2}$

\section{Climate change litigation und Klimaklagen weltweit}

In diesem Zusammenhang ist die erste erfolgreiche Klimaklage der niederländischen NGO Urgenda zu nennen, die von einem Gericht in Den Haag 2015 positiv beschieden wurde. Das Gericht entschied, dass der niederländische Staat seine Bemühungen in Bezug auf den Klimaschutz erhöhen und seine THG-Emissionen bis 2020 um mindestens 25\% im Vergleich zu 1990 senken müsse. ${ }^{3}$ Sowohl die Regierung als auch Urgenda legten Berufung gegen das Urteil ein. Das Gericht erster Instanz hatte noch argumentiert, dass Urgenda als juristische Person nicht in ihren Menschenrechten verletzt und sich somit gemäß Art. 34 der Europäischen Menschenrechtskonvention (EMRK) nicht auf die Konventionsrechte berufen könne. Das Berufungsgericht verwarf 2018 diese Argumentation und erklärte, dass Art. 34 EMRK nur die Voraussetzungen für das Verfahren vor dem EGMR regele. Im Übrigen hielt es das Urteil und damit die Verpflichtung zur Reduzierung der Emissionen um mindestens 25\% aufrecht. Hierzu stützte es sich auf das Recht auf Leben (Art.2) und das Recht auf Privatleben und Familie (Art. 8) aus der EMRK, welche die positive Verpflichtung beinhalten, die Bürger:innen vor den negativen Folgen des Klimawandels zu schützen. ${ }^{4}$ Dieses Urteil wurde 2019 letztinstanzlich vom Hohen Rat der Niederlande bestätigt. ${ }^{5}$

In den USA sind bisher die meisten Klimaklagen angestoßen worden. ${ }^{6}$ Im Jahr 2015 haben 21 Kinder und Jugendliche die Regierung der USA dahingehend beschuldigt, von den Gefahren der Öl- und Gasverbrennung jahrzehntelang gewusst und diese bewusst verschwiegen zu haben.

2 Statement der United Nations High Commissioner for $\mathrm{Hu}$ man Rights, Michelle Bachelet, https://ohchr.org/EN/NewsEvents/Pages/DisplayNews.aspx?NewsID=26343\&LangID=E (letzter Aufruf am 25.3.2021).

3 District Court of The Hague, Urgenda $v$ The Netherlands (ECLI:NL:RBHDHA:2015:7196), 2015, im Folgenden: Urgenda I.

4 The Hague Court of Appeal, Appeal Decision Urgenda $v$ The Netherlands (ECLI:NL:GHDHA:2018:2610), 2018, Rn. 41-43, im Folgenden: Urgenda II.

5 Supreme Court of the Netherlands, Urgenda $v$ The Netherlands (ECLI:NL:HR:2019:2007), 2019.

6 Gemäß des Sabin-Center for Climate Change Law der Columbia University New York waren es im März 20211350 Klagen (anhänging und entschieden), http://cli matecasechart.com/us-climate-change-litigation/ (letzter Aufruf am 25.3.2021) 
Dadurch habe sie die Zukunft der jungen Menschen aufs Spiel gesetzt und ihr Recht auf ein stabiles Klima verletzt. ${ }^{7}$ Sechs Jahre später ist die Zulässigkeit der Klage immer noch nicht abschließend geklärt. Es ist fraglich, ob die Kläger:innen angesichts eines fehlenden Grundrechts auf eine gesunde Umwelt klagebefugt sind und ob ein gerichtliches Urteil ihrer Beschwer überhaupt abhelfen kann. Richterin Ann Aiken hatte 2015 zunächst erklärt, dass es ein Recht auf ein stabiles Klima gibt:

"I have no doubt that the right to a climate system capable of sustaining human life is fundamental to a free and ordered society. Just as marriage is the 'foundation of the family', a stable climate system is quite literally the foundation 'of society, without which there would be neither civilization nor progress." "

Dieses Recht sei so grundlegend, dass es nicht einmal in die Verfassung geschrieben sein muss, um seine Existenz anzuerkennen. Aufgrund einer möglichen Verletzung des Fünften Zusatzartikels der US-amerikanischen Verfassung, aus dem Aiken dieses Recht herleitete, hielt sie die Kläger:innen für klagebefugt und ließ die Klage zu. Die Regierung bestreitet, dass ein „Recht auf ein Klimasystem, das dazu fähig sei, menschliches Leben aufrechtzuerhalten" existiert und forderte, die Klage als unzulässig abzuweisen. In der Folge versuchte sie durch mehrere Handlungen eine Hauptverhandlung des Falles zu verhindern.9 Am 17. Januar 2020 lehnte der Ninth Circuit Court of Appeals die Klage schließlich als unzulässig ab. ${ }^{10}$ Die Kläger:innen haben Beschwerde gegen diese Entscheidung eingelegt. Obwohl das Verfahren noch in der Frage der Zulässigkeit steckt, so hat es doch immense Aufmerksamkeit erregt. Mehr als 25 amicus briefs sind im Laufe des Verfahrens eingegangen. ${ }^{11}$ Das ungewöhnliche Verhalten der Regierung (die Stellung eines dritten „writ of mandamus“-Antrag ist au-

7 Vgl. die Klageschrift: First Amendment Complaint for Declaratory and Injunctive Relief (No. 6:15-cv-01517-TC), Kelsey Cascadia Rose Juliana et al. $v$ United States, 2015, Rn. 1.

8 District Court of Oregon, Judge Ann Aiken, Kelsey Cascadia Rose Juliana v United States of America (Case No. 6:15-cv-01517-TC), 11 October 2016, S. 32, im Folgenden: Juliana.

9 Zwei Eingaben an den Obersten Gerichtshof der Vereinigten Staaten und drei sog. „writ of mandamus“-Anträge, womit sie den Fall als unzulässig erklären lassen wollte.

10 S. u. Fn. 42.

11 Der Verfahrensgang und entsprechende Dokumente können in der Datenbank des Sabin Center unter http://climatecasechart.com/case/juliana-v-united-states (letzter Aufruf am 30.4.2021) abgerufen werden. 
ßerordentlich selten) lässt darauf schließen, dass die US-Regierung den Fall sehr ernst nimmt und eine gerichtliche Entscheidung zu mehr Klimaschutz unter allen Umständen verhindern möchte.

Unter den Begriff „climate change litigation“ fallen rechtliche Verfahren, die das Ziel haben, klimafreundliches Verhalten zu erzwingen. Im Gegensatz zu „klassischen“ Prozessen handelt es sich hierbei oftmals nicht um Einzelfälle, in denen es den Kläger:innen tatsächlich um die Beseitigung einer Rechtsverletzung geht, sondern um strategische Prozessführung, die darauf gerichtet ist, ein bestimmtes gesetzgeberisches oder soziales Verhalten herbeizuführen. ${ }^{12}$ Dabei können sich diese Verfahren sowohl gegen Regierungen als auch Unternehmen richten, die dadurch in die Verantwortung für einen hohen THG-Ausstoß genommen werden sollen. In Deutschland sorgte der Fall des peruanischen Kleinbauern und Bergführers Saúl Luciano Lliuya, der eine Klage gegen RWE eingelegt hat, für Aufsehen. Lliuya fordert von RWE, sich an den Reparaturkosten eines Staudamms zu beteiligen, der einen See, dessen Wasserstand aufgrund der klimabedingt immer schnelleren Gletscherschmelze kontinuierlich steigt, davon abhalten soll, seine Heimatstadt Huaraz in den Anden zu überfluten. ${ }^{13}$ Während das LG Essen die Klage als „Rechtssache von grundlegender Bedeutung" eingestuft, aber letztlich abgewiesen hatte, hat das OLG Hamm als Berufungsgericht eine grundsätzliche Unternehmenshaftung für Klimaschäden zunächst bejaht. ${ }^{14}$

Im Folgenden soll es nur um solche Klimaklagen gehen, die sich gegen den Gesetzgeber selbst richten. Hier stellt sich die grundsätzliche Frage, ob ein Gericht diesen mittels Urteils zu mehr Klimaschutz verpflichten kann, wenn er nach dessen Auffassung zu wenig gegen den Klimawandel unternimmt.

\section{Der Klimawandel als polyzentrisches Problem}

Das Recht auf eine gesunde Umwelt ist in vielen nationalen Verfassungen und Menschenrechtskonventionen (z.B. in Art. 24 der Afrikanischen

12 Peel/Osofsky, Climate Change Litigation: Regulatory Pathways to Cleaner Energy, 2015, S. 33.

13 DW, https://p.dw.com/p/3UF1w (letzter Aufruf am 25.3.2021).

14 Vgl. den Hinweis- und Beweisbeschluss des OLG Hamm v. 30.11.2017, Saúl Luciano Lliuya ./. RWE AG, Rn. 2. Aktenzeichen; Fundstelle Der Ortstermin zur Beweisaufnahme musste aufgrund der Corona-Pandemie aber bis auf Weiteres verschoben werden. 
Charta der Menschenrechte und Rechte der Völker) festgeschrieben. Ein solches Recht, soweit es justiziabel ist, kann der Einzelnen helfen, gegen Umweltzerstörungen vorzugehen.

Der EMRK und der US-amerikanischen Verfassung ist so ein Recht unbekannt. Stattdessen haben die Gerichte durch eine dynamische Interpretation „klassischer" Grundrechte ökologische Belange berücksichtigt. Das Phänomen der richterlichen Rechtsfortbildung ist auch in anderen Rechtsbereichen, die einem schnellen Wandel unterliegen (z.B. Datenschutzrecht) nicht neu. ${ }^{15}$ Allerdings waren Richter:innen früher eher vorsichtig, wenn ein Sachverhalt Umweltbezug hatte und haben öfter noch entschieden, dass Umweltschutz - und damit der Schutz eines Allgemeingutes - nicht Gegenstand eines Gerichts-, sondern eines Gesetzgebungsverfahrens sein müsse. ${ }^{16}$ Das hat sich gewandelt. Unter dem Stichwort des "greening“" werden insbesondere Grund- und Menschenrechte „ökologisch“ interpretiert. ${ }^{17}$ Der Gesetzgeber kann durch das Unterlassen von Umweltschutz seine menschenrechtlichen Schutzpflichten verletzen, obwohl die Umwelt selbst nicht Gegenstand einer menschenrechtlichen Norm ist.

Besonders deutlich wird das mit Blick auf die EMRK. Die Bemühungen um ein Zusatzprotokoll, mit dem ein Menschenrecht auf gesunde Umwelt etabliert werden sollte, scheiterten in den 1970ern. ${ }^{18}$ Nichtsdestotrotz hat sich der EGMR insbesondere auf Art. 2 (Recht auf Leben) und Art. 8 (das Recht auf Privatleben und Familie) berufen, um positive Pflichten der Mitgliedsstaaten hinsichtlich besseren Schutzes von Umwelteinflüssen zu begründen. ${ }^{19}$ Von Umweltproblemen, die zunächst nur Einzelne betrafen, hat sich die Rechtsprechung in den letzten Jahren durch die angesprochenen Klimaklagen dahingehend weiterentwickelt, dass auch ein globales Problem, das potentiell alle Menschen betreffen kann, solche Schutzpflichten auslösen kann. ${ }^{20}$ Das kann dazu führen, dass ein vom Gesetzgeber beschlossenes Klimaziel - wie im Fall der Niederlande, die eine Emissionsreduzierung von 14-17\% angestrebt hatten - von einem Gericht

15 Vgl. Busch/Jakobi, in: Hönnige/Kneip/Lorenz, Verfassungswandel im Mehrebenensystem, 2011, S. $297 \mathrm{ff}$.

16 Vgl. Benjamin, Pace Environmental Law Review 2011, 582 (583 ff).

17 Albers, Security and Human Rights 2017, 113 (121 ff.).

18 Mileva/Fortuna, in: Abi-Saab u.a. (Hrsg.), Evolutionary Interpretation and International Law, 2019, S. 123 (131).

19 Eine Übersicht findet sich im Manual on Human Rights and the Environment des Council of Europe, 2012.

20 Vgl. die Beschwerde der portugiesischen Jugendlichen vor dem EGMR, Youth for Climate Action v Austria, et al, 2020. 
als nicht ambitioniert genug verworfen wird. ${ }^{21}$ Teilweise versuchen Kläger:innen durch strategische Prozessführung neue Menschenrechte, wie das Recht auf ein stabiles Klima ${ }^{22}$, ein Recht auf Zukunft $^{23}$ oder Umweltrechte für zukünftige Generationen ${ }^{24} \mathrm{zu}$ schaffen.

Von Umweltaktivist:innen wird das progressive Vorangehen der Gerichte bejubelt. Allerdings wird das Instrument der strategischen Prozessführung durchaus kritisch gesehen, da versucht werde, im Namen des allgemeinen öffentlichen Interesses Partikularinteressen durchzusetzen. ${ }^{25}$ Gerichte würden Klimapolitik betreiben, wenn sie dem Klimaschutz den Vorrang vor anderen (z.B. wirtschaftlichen) Interessen geben würden. ${ }^{26}$

Es stellt sich dabei die Frage, ob Gerichte „klassische“ ökologische Aspekte unter den Grund- oder Menschenrechtsschutz fassen dürfen, wenn doch die Problematik des Klimawandels bei Abschluss der völkerrechtlichen Verträge noch nicht bekannt war. Das ist zunächst eine Methodenfrage - dürfen Gerichte so „fortschrittlich“ interpretieren? Gleichzeitig hat diese Frage aber auch Auswirkungen auf andere Bereiche des demokratischen Rechtsstaats, insbesondere die Frage der Gewaltenteilung. Die effektive Beseitigung von Rechtsverletzungen, die im Zusammenhang mit dem Klimawandel stehen, ist nur durch weitgehende Maßnahmen möglich durch rigide Regelungen, die die Auswirkungen des Klimawandels begrenzen („mitigation“), durch Anpassung an veränderte klimatische Bedingungen („adaptation“) oder eine Kombination aus beiden. ${ }^{27}$ Solche Maßnahmen begünstigen dann aber nicht nur die Person, die erfolgreich klagt, sondern haben vielmehr Auswirkungen auf die gesamte Gesellschaft. Verglichen mit den „einfach“ gelagerten Streitigkeiten des Umweltschutzes wird hier die Reichweite des Problems gerichtlichen Rechtsschutz gegen den Klimawandel deutlich. Während z.B. eine Entscheidung über den Fortbetrieb einer toxischen Deponie in der unmittelbaren Nachbarschaft eines Wohnhauses nur die Anwohner:innen der verschmutzenden Anlage und deren Betreiberin betrifft - sich also oft im Rahmen eines klassischen

21 Urgenda I (Fn. 3), Rn. 4.26.

22 Juliana (Fn. 8), S. 32.

23 Verfassungsbeschwerde Neubauer u.a. (AZ: 1 BvR 288/20), eingereicht am 12.2.2020.

24 Corte Suprema de Justicia de Colombia, Demanda Generaciones Futuras v. Minambiente (STC 4360-2018), 2018, Rn. 5.3, im Folgenden: Future Generations.

25 Hahn, Zeitschrift für Rechtssoziologie 2019, 5 (15, 18).

26 Burgers, Transnational Environmental Law 2020, 55 ( $57 \mathrm{ff}$ ).

27 Intergovernmental Panel on Climate Change, Climate Change - Impacts, Adaptation, and Vulnerability (Working Group II, Summary for Policymakers), 2014. 
„Zwei-Parteien“-Rechtsstreit abspielt -, betreffen Klimaklagen, die sich gegen den Staat richten, eine große Anzahl an Menschen, mitunter die gesamte Bevölkerung. ${ }^{28}$ Schließlich verursacht nicht der Staat selbst die Emissionen, sondern alle Bürger:innen in ihrer Stellung als Autobauer:innen, Energielieferant:innen, Landwirt:innen oder Konsument:innen. Ein konsequentes Vorgehen gegen den Klimawandel ist daher ohne Einschnitte in berufliches oder privates Verhalten anderer nicht möglich.

Der Klimawandel ist daher ein polyzentrisches Problem - aufgrund seiner Komplexität liegen viele verschiedene Zentren vor, die in Spannung zueinanderstehen. Fuller vergleicht diese polyzentrischen Fälle mit einem Spinnennetz: das Ziehen an einem Faden hat Auswirkungen auf ein anderes Zentrum und schlussendlich auf das gesamte Gebilde. ${ }^{29}$ In solchen Situationen stößt die Rechtsprechung mit ihren Möglichkeiten an ihre Grenzen, da sie nur den Fall vor sich bearbeiten, aber nicht die Auswirkung auf die anderen „Fäden“ beeinflussen kann. Statt eines Urteils bedarf es in solchen komplexen Situationen eines "politischen Deals“ im Parlament, der auf Verhandlungen und Kompromisse setzt und somit die Belange aller Betroffenen miteinbeziehen kann. ${ }^{30}$ Wenn also Gerichte über klimapolitische Fragen entscheiden, so der Vorwurf, dann würden sie sich in die Kompetenzen der Legislative einmischen und unerlaubt Klimapolitik betreiben.

\section{Gewaltenteilung und Demokratie}

Die Gewaltenteilung in Legislative, Exekutive und Judikative ist ein vitales Element des demokratischen Rechtsstaats. Sie geht zurück auf Montesquieu, der mit der Teilung die Tyrannei durch den Monarchen, der alle Gewalten in einer Hand hält, zu vermeiden suchte. ${ }^{31}$

Stattdessen liegt die Staatsgewalt in der Republik in der Hand des Volkes, ausgeübt wird sie aber von den Staatsorganen. Ihre Ausübung kann durch drei Arten der demokratischen Legitimation gerechtfertigt werden. ${ }^{32}$ Zum einen sind in der Verfassung, die das Volk sich gegeben

28 Zürn, Government and Opposition 2004, 260 (269).

29 Fuller, Harvard Law Review 1978, 353 (395 ff).

30 Fuller (Fn. 29), 400.

31 Montesquieu, Complete Works, Vol. 1 (The Spirit of Laws, Bd. 1, 1748), 1777, S. 198 ff.; Martinez, in: Rosenfeld/Sajó (Hrsg.), The Oxford Handbook of Comparative Constitutional Law, 2012, S. 548.

$32 \mathrm{Zu}$ alledem s. Göpl, Staatsrecht I, 12. Aufl. 2020, Rn. $262 \mathrm{ff}$. 
hat, die Organe selbst und ihre Aufgaben festgeschrieben (institutionellfunktionelle Legitimation). Zudem wird nach der Enge der Bindung an den Volkswillen differenziert. Das Parlament ist das einzige Organ, welches direkt von der (wahlberechtigten) Bevölkerung gewählt wird und damit unmittelbar legitimiert ist. Die Legitimationskette ist besonders kurz und stark. Die Mitglieder der Exekutive und Judikative hingegen sind nur mittelbar durch das Parlament legitimiert (organisatorisch-personelle Legitimation). Diese müssen sich auch nicht regelmäßig zur Wahl stellen - und somit dem Volk nicht persönlich Rede und Antwort stehen. Umgekehrt bedeutet dies, dass „Richter an Recht und Gesetz gebunden [sind], nicht an die Wünsche und Gefühle des Volkes." ${ }^{33}$ Daneben verlangt die sachlich-inhaltliche Legitimation, dass die Parlamente Inhalt, Umfang und Grenzen der Kompetenzen der einzelnen Organe bestimmen.

Insofern wird das Parlament als das „Willensorgan“ angesehen - es verkörpert und spiegelt den Willen des Volkes, welcher in Gesetzesform gegossen wird. Die Exekutive und Judikative hingegen, so hieß es lange Zeit, seien nur zur Anwendung eben jener Gesetze ermächtigt, ohne aber selbst zur Schaffung von Recht befugt zu sein. ${ }^{34}$

Aber diese Argumentation hat ihre Schwachstellen. Sie hat ihren Ausgangspunkt in einem rein nationalstaatlichen Kontext: nach dem bekannten liberalen Gedanken soll man nur den Regelungen, denen man (mittelbar durch seine Repräsentant:innen) auch zugestimmt hat, unterworfen sein. ${ }^{35}$ Problematisch wird es aber bei Angelegenheiten, die sich wenig um Staatsgrenzen scheren, wie etwa dem Klimawandel. Deutsche Emissionen bleiben schließlich nicht in Deutschland, sondern vermischen sich in der Atmosphäre und erzeugen bereits heute stärkere und häufigere Naturkatastrophen wie Dürren, Fluten, Stürme in weit entfernten Ländern. Damit haben Entscheidungen über den Betrieb eines besonders emissionsreichen Kohlekraftwerks in Deutschland auch Auswirkungen für die Menschen auf Fidschi, die um den Erhalt ihrer Heimat bangen müssen. Entscheidungen über die Reduzierung (oder Nicht-Reduzierung) von Emissionen haben also Wirkung über die eigenen Grenzen hinaus.

33 Max-Planck-Gesellschaft, https://mpg.de/13895994/unabhaengige-richter (letzter Aufruf am 25.3.2020).

34 Möllers, in: Kreide/Niederberger, Transnationale Verrechtlichung, 2008, S. 160 (174 ff).

35 Habermas, Faktizität und Geltung. Beiträge zur Diskurstheorie des Rechts und des demokratischen Rechtsstaats, 1992, S. 138: „Gültig sind genau die Handlungsnormen, denen alle möglicherweise Betroffenen als Teilnehmer an rationalen Diskursen zustimmen konnten.“ 
Hier stellen sich tiefgreifende Fragen der Legitimität, denn die Menschen in betroffen(er)en Ländern können in Deutschland nicht wählen und haben somit keine Möglichkeit der Einflussnahme im nationalen Willensbildungsprozess.

Dürfen Staaten in den Kontexten der ,justice-senstive externalities“36 wirklich nur das Wohl der eigenen Bevölkerung im Auge haben? Zwar war es lange Konsens, dass jeder Staat einzig und allein seinem eigenen Volk gegenüber positive, auch finanzielle, Verpflichtungen hat. Aber die Problematik des Klimawandels rückt auch Gerechtigkeitsfragen in den Mittelpunkt, die tief mit der Legitimationsfrage verknüpft sind. Schließlich sind es die Länder des Globalen Nordens, die die meisten Emissionen verursachen, aber die Länder des Globalen Südens, die die Konsequenzen tragen müssen. ${ }^{37}$ Einige argumentieren daher, dass in solchen Fällen der Staat nicht unilateral Entscheidungen treffen darf, die die Interessen der Bevölkerung anderer Staaten so einschneidend verletzen. Vielmehr müssen Staaten hier als „trustees of humanity“ auftreten, als Stellvertreter der gesamten Menschheit. ${ }^{38}$

Die gleiche Frage stellt sich für zukünftige Generationen, denn ungeborene oder sehr junge Menschen können oder dürfen nicht wählen. Sie werden aber in viel stärkerem Maße betroffen sein als jetzige Generationen, da man annimmt, dass sich die volle Wucht des Klimawandels erst in den nächsten Jahrzehnten entfalten wird. „The climate crisis is a children's rights crisis“, heißt es dazu in einer Beschwerde gegen fünf Staaten, darunter Deutschland, die von Kindern und Jugendlichen 2019 beim UN-Ausschuss für die Rechte des Kindes eingereicht worden ist. ${ }^{39}$

Klimaklagen sind insofern auch ein Vehikel für Menschen, deren Stimmen in den Parlamenten ungehört bleiben. Denn in Verfahren können auch diejenigen Partei sein, die (noch) nicht wählen dürfen und ihre Argumente sowohl dem Gericht als auch der breiten Öffentlichkeit bekannt machen. ${ }^{40}$ So wurde eine der Verfassungsbeschwerden gegen die Bundes-

36 Zum Konzept der ,justice-sensitive externalitites“: Kumm, Indiana Journal of Global Legal Studies 2013, 605 (613 ff).

37 Siehe dazu z. B. Gonzalez, in: Kotzé (Hg.), Environmental Law and Governance for the Anthropocene, 2017, 219-40. Im Jahr 2007 stieß die/der durchschnittliche US-Amerikaner:in so viele THG aus wie über 500 Bewohner:innen in Äthiopiens, Tschad, Afghanistan, Kambodscha oder Burundi, vgl. Malm/Hornborg, Anthropocene Review 2014, 62 (64).

38 Dazu Benvenisti, AJIL 2013, 295.

39 Sacchi/Lorenzo u.a., Communication to the Committee on the Rights of the Child, 2019, S. 3.

40 Lahav, In Praise of Litigation, 2017, S. 85. 
republik von Kindern und Jugendlichen aus Deutschland und Menschen aus Nepal und Bangladesch eingereicht. Auch viele andere Klimaklagen weisen einen intergenerationellen Zusammenhang auf. ${ }^{41}$ Klimaklagen eröffnen hier den Zugang zu Recht - und zu Gerechtigkeit.

Diese Klagen werden bewusst eingesetzt, um ein bestimmtes parlamentarisches Verhalten durch das Gericht zu erzwingen, sie berühren also willentlich - politische Belange und damit Kernkompetenzen der Legislative. Müssten Gerichte sich also von vornherein für unzuständig in Sachen Klimaklagen erklären? Der US-amerikanische Ninth Circuit Court of Appeals scheint das in seinem Juliana-Urteil so zu sehen. Das Gericht führt in seiner Entscheidung hinsichtlich der Unzulässigkeit der Klage aus, die gewünschte Entscheidung der Kläger:innen

„would necessarily require a host of complex policy decisions entrusted to the wisdom and discretion of the executive and legislative branches. The panel reluctantly concluded that the plaintiffs' case must be made to the political branches or to the electorate at large. "42

Die Frage der Gewaltenteilung hat auch die Gerichte beschäftigt, die Klimaklagen positiv beschieden haben. In der Sache Urgenda führte das erstinstanzliche Gericht aus, dass es in den Niederlanden keine vollumfängliche Gewaltenteilung gebe, sondern es vielmehr ein Gleichgewicht zwischen den Gewalten geben müsse. ${ }^{43}$ Zurückhaltung ist dann geboten, wenn es um die politische Abwägung unterschiedlicher Interessen gehe, die Auswirkungen auf die Struktur oder Organisation der Gesellschaft haben könne. Das Gericht wies jedoch auch darauf hin, dass es ein wesentliches Merkmal der Rechtsstaatlichkeit sei, dass Handlungen politischer Organe von unabhängigen Gerichten beurteilt werden können und müssen. Denn es sei gerade ein Akt der Legislative gewesen, der die Gerichte zur Entscheidung in Rechtsstreitigkeiten ermächtigt habe. ${ }^{44}$

Das Berufungsgericht und das Verfassungsgericht haben ihre Entscheidungen v.a. auf die positiven Verpflichtungen aus Art. 2 und 8 der EMRK gestützt. Der Hauptkritikpunkt an der Anwendung der Doktrin der positiven Verpflichtungen auf den Klimawandel ist ihr unklarer Anwendungsbereich. Positive Verpflichtungen können auf viele Arten und Weisen erfüllt werden und erfordern die Abwägung und Priorisie-

41 Vgl. u.a. die Youth for Climate Action v Austria, et al, 2020.

42 Ninth Circuit Court of Appeals, Juliana v United States (No. 18-36082), 17.1.2020, S. 5 .

43 Urgenda I (Fn. 3), Rn. 4.95.

44 UrgendaI (Fn. 3), Rn. 4.97. 
rung zwischen konkurrierenden Interessen. ${ }^{45}$ Daher beinhalten sie eine zutiefst politische Komponente, die von den Gerichten unangetastet bleiben muss. Es stellt sich damit die Frage, ob die Doktrin von den positiven Verpflichtungen zumindest auf komplexe Fälle, die sich von dem ursprünglichen Kontext unterscheiden, für den sie entwickelt wurde - Schäden, die aus einer einzigen Quelle resultieren -, nicht angewendet werden sollte. ${ }^{46}$ Allerdings erscheint es geradezu paradox, warum ein Gericht nicht über eine Gefahr entscheiden sollte, die weit mehr Menschen bedroht und potenziell gefährlicher ist als lokal-begrenzte Gefahren. In solchen Fällen ist doch eher mehr denn weniger Schutz erforderlich. ${ }^{47}$

Das Berufungsgericht setzte sich mit dieser Frage nicht auseinander, sondern nahm unter Bezugnahme auf die klimawissenschaftlichen Erkenntnisse eine Verletzung von Art. 2 und 8 EMRK an. ${ }^{48}$ Das Gericht betonte, dass es verpflichtet sei, für die Niederlande verbindliches internationales Recht anzuwenden, da dieses Teil der niederländischen Jurisdiktion sei und sogar Vorrang vor nationalem Recht genieße. ${ }^{49}$ Es betonte aber auch, dass es kein neues Gesetzesrecht schaffen wolle (was nach niederländischem Recht verboten ist). Es könne somit nur eine Verletzung positiver menschenrechtlicher Verpflichtungen feststellen, muss es aber ins Ermessen der anderen Gewalten stellen, die Maßnahmen zur Beseitigung der Verletzung zu wählen. Der Ermessensspielraum für den Staat ist insofern begrenzt, als dass er nicht entscheiden kann, das Problem nicht anzugehen (das $\mathrm{Ob}$ ). Er hat aber einen Ermessensspielraum, wie er das Ziel der 25\%igen THG-Reduzierung erreichen will..$^{50}$

Jüngst hat das deutsche BVerfG das Klimaschutzgesetz von 2019 für teilweise verfassungswidrig erklärt, da dieses zwar bis 2030 Einsparziele in verschiedenen Bereichen vorsieht, aber offen gelassen hat, welche Einsparungen für den Zeitraum 2031 bis 2050 verfolgt werden müssen oder wie sie erreicht werden sollen. ${ }^{51}$ Dies gehe zu Lasten der nachfolgenden Generationen, deren Freiheiten durch das heute übers Maß lebenden Generationen eingeschränkt werden. Das BVerfG hat insbesondere anerkannt, dass der demokratisch politische Prozess über Wahlperioden kurzfristiger

45 Leijten, Netherlands Quarterly of Human Rights 2019, 112 (116f).

46 Burgers/Staal, Amsterdam Law School Legal Studies Research Paper No. 2019-01, 2019, S. 6.

47 Burgers/Staal (Fn. 46), S. 14.

48 Urgenda II (Fn. 4), Rn. 44 f.

49 Urgenda II (Fn. 4), Rn. 69.

50 Urgenda II (Fn. 4), Rn. 68, 74.

51 BVerfG, B. v. 24.3.2021 - 1 BvR 2656/18. 
organisiert ist und es insofern umso schwerer fällt, langfristig Klimaziele $\mathrm{zu}$ verfolgen, v.a. weil die betroffenen zukünftigen Generationen keine Stimme in diesem Prozess haben. ${ }^{52}$ Insofern versteht das Gericht Art. 20a GG als „eine justiziable Rechtsnorm, die den politischen Prozess zugunsten ökologischer Belange auch mit Blick auf die besonders betroffenen künftigen Generationen binden soll." ${ }^{53}$ Diese Bindung drohe verloren zu gehen, wenn der materielle Gehalt des Art. 20a GG nur im tagespolitischen Prozess entschieden würde. Zwar lasse Art. 20a GG dem Gesetzgeber erheblichen Spielraum. Aber die Grundrechte schützen subjektivrechtlich als „intertemporale Freiheitssicherung“ vor einer einseitigen Verlagerung von Klimaschutzmaßnahmen in die Zukunft. ${ }^{54}$ Damit trägt das Gericht zu einer nachhaltigeren Demokratie bei, in der auch die Freiheiten der zukünftigen Anderen berücksichtigt werden müssen. Das könnte künftig gar für ausländische Betroffene gelten. Das BVerfG hielt es für „prinzipiell denkbar", dass Schutzpflichten auch gegenüber den in Bangladesch und in Nepal lebenden Beschwerdeführenden bestehen und Deutschland verpflichten, gegen Beeinträchtigungen durch den globalen Klimawandel vorzugehen. ${ }^{55}$

\section{Rechtsprechung als Rechtserzeugung?}

Diese Fälle zeigen deutlich, dass die Annahme, wonach Gerichte ausschließlich Recht anwenden, welches zuvor von der Legislative erzeugt wurde, nicht trägt. Das ist kein klima-rechtliches Novum: so ist schon die Doktrin von (europarechtlichen) positiven Verpflichtungen eine Schöpfung der Judikative. ${ }^{56}$ Gleiches lässt sich über eine Reihe von Persönlichkeitsgrundrechten sagen, insbesondere dem Grundrecht auf informationelle Selbstbestimmung, welches das BVerfG durch seine Rechtsprechungspraxis geschaffen hat. Zwar haben viele Jurist:innen rigoros bestritten, dass das höchste Gericht ein neues Grundrecht erfunden, sondern nur herausgearbeitet hätte, was ohnehin schon „angelegt“ war. Schlink stellt

52 BVerfG, 1 BvR 2656/18, Rn. 206.

53 BVerfG, 1 BvR 2656/18, LS 1 a.

54 BVerfG, 1 BvR 2656/18, Rn. 183.

55 BVerfG, 1 BvR 2656/18, Rn. 174 ff. Das Gericht hatte zuvor bereits eine Verletzung der Schutzpflichten der inländischen Beschwerdeführenden abgelehnt und musste sich mit der Frage nicht auseinandersetzen, betonte aber, dass diese zumindest von anderer Art wären als Schutzpflichten gegenüber Inländer:innen.

56 Krieger, ZaöRV 2014, 187 (189). 
jedoch zutreffend fest, dass „der Unterschied zwischen der Erfindung neuer Grundrechte, der Entdeckung neuer Grundrechtsbedeutungen und der neuen Beleuchtung alter Grundrechtswirkungen vernachlässigt werden [kann]. Er belegt denselben Vorgang richterlicher Rechtsschöpfung nur mit verschiedenen Begriffen. " 57 Das Recht auf informationelle Selbstbestimmung, aber daneben auch weiteres „ungeschriebenes Verfassungsrecht" wie u.a. das materielle Rechtsstaatsprinzip, das Bestimmtheitsgebot, der Verhältnismäßigkeitsgrundsatz, sind größtenteils das Produkt höchstrichterlicher Interpretation - und damit Rechtsschöpfung. ${ }^{58}$

Eine Norm kann nicht für jede erdenkliche Situation die einzig richtige Rechtsfolge aufstellen. So klar und eindeutig sie auch erscheinen mag, eine Norm muss immer interpretiert werden, bevor sie angewendet werden kann. „Es gibt keine Rechtsnormen, es gibt nur interpretierte Rechtsnormen!" 59 Man kann die Norm mit einer leeren Schale vergleichen, die erst durch Interpretation gefüllt wird. Jeder noch so eindeutig formulierte Rechtsakt lässt der Rechtsanwenderin einen Ermessenspielraum. ${ }^{60}$ Kelsen legt insofern dar, dass Interpretation mittels der gängigen Interpretationsmethoden in einem konkreten Fall nicht die eine richtige Entscheidung liefern kann, sondern dass sie vielmehr den Rahmen der Norm herstellt, in dem mehrere Alternativen denkbar sind. ${ }^{61}$ Je vager die Norm ausgestaltet ist, desto mehr ist die konkrete Bestimmung des Inhalts auf den Anwendungsfall in das Ermessen der Richterin gestellt. Das gilt insbesondere bei Generalklauseln. ${ }^{62}$ Es folgt, dass „the open texture of law leaves a vast field for a creative activity which some call legislative." ${ }^{63}$ In diesem weiten Feld spielen nicht nur die Normen des positiven Rechts, sondern auch solche der Moral, Gerechtigkeit und sozialen Werturteile (Volkswohl, Staatsinteresse usw.) eine Rolle. ${ }^{64}$ Die authentische Interpretation - also die Interpretation durch ein rechtsanwendendes Organ - schafft Recht. ${ }^{65}$

Das gilt nicht nur für die Interpretation, die in Form eines Gesetzes ergeht und somit für alle gleichen Fälle Recht schafft - also den klassische

57 Schlink, Der Staat 25 (1986), 233.

58 S. zur Thematik Wolff, Ungeschriebenes Verfassungsrecht unter dem Grundgesetz, 2000, S. $3 \mathrm{ff}$.

59 Häberle, ZfP 1974, 111 (127).

60 Kelsen, Reine Rechtslehre, 2. Aufl. 1960, S. 348 f.

61 Kelsen, Rechtslehre (Fn. 60), S. 349.

62 Payandeh, Judikative Rechtserzeugung, 2017, S. 322.

63 Hart, The Concept of Law, 1961, S. 204.

64 Kelsen, Rechtslehre (Fn. 60), S. 351.

65 Kelsen, Rechtslehre, (Fn. 60), S. 352. 
Rechtssetzungsakt durch den Gesetzgeber, der seinerseits bei der Erzeugung einfachen Rechts Normen, z.B. jene der Verfassung, interpretiert sondern auch für die Interpretation, die nur in einem konkreten Einzelfall eine Norm erzeugt. Auch wenn das Gericht weniger frei ist als der Gesetzgeber: Durch authentische Interpretation setzen - insbesondere die letztinstanzlichen - Gerichte neues Recht, sobald diese Akte rechtskräftig geworden sind. ${ }^{66}$ Rechtsvollziehung ist auch Rechtserzeugung, zwischen beiden lässt sich nicht eindeutig differenzieren. Merkl prägte hierfür den Begriff des „doppelten Rechtsantlitz“. ${ }^{67}$ Damit muss das klassische Verständnis von Gewaltenteilung - die Legislative „will“, die Judikative „handelt“ ohne einen persönlichen Beitrag zu dieser Ausführung zu leisten - überdacht werden. Möllers regt insofern an, die Aufgabe der Gewalten nicht in der Ausübung von Macht, sondern in der Erzeugung von Recht zu sehen. ${ }^{68}$

Dann stellt sich aber die Frage, worin eigentlich der Unterschied zwischen den Gewalten liegt und wie man ihre Kompetenzen voneinander abgrenzen soll. Es gilt, dass der Grundsatz der Gewaltenteilung im Kern gewahrt bleiben muss. Möllers greift hierfür auf Art und Umfang der Rechtserzeugung zurück. Entscheidend für sein Modell ist das Element der Selbstbestimmung. Jeder Rechtssetzungsakt - gleichgültig, ob er von der Legislative, Exekutive oder Judikative stammt - muss demokratisch und individuell legitimiert sein. Er teilt hier zwischen demokratischer und individueller Selbstbestimmung, die gleichranging sind. ${ }^{69}$ Die demokratische Selbstbestimmung, die der Legislative zugeordnet wird, steht für die Regelung einer Vielzahl von Sachverhalten für eine Vielzahl von Menschen in einem in die Zukunft gerichteten Prozess. Diesen Prozess kann die Legislative von sich aus anstoßen, durch die Einleitung eines Gesetzgebungsverfahrens, in das die Gerichte grundsätzlich nicht eingreifen dürfen. Die Judikative, verantwortlich für die individuelle Selbstbestimmung, kann hingegen nur auf fremde Initiative tätig werden. Der ganze Prozess wird vom Willen der Klägerin getragen. Der Prozess ist grundsätzlich in die Vergangenheit gerichtet, also auf die Wiedergutmachung bereits entstandener Schäden. ${ }^{70}$

66 Kelsen, Rechtslehre, (Fn. 60), S. 352.

67 S. Merkl, Juristische Blätter 1918., Rn. $425 \mathrm{ff}$.

68 Möllers, Die drei Gewalten. Legitimation der Gewaltengliederung in Verfassungsstaat, Europäischer Integration und Internationalisierung, 2008, S. 90.

69 Möllers, Gewalten (Fn. 68), S. 73.

70 Möllers, Gewalten (Fn. 68), S. 95-106. 
Während also beide Gewalten ${ }^{71}$ Recht schaffen, so sind die Rechtsetzungsverfahren unterschiedlich und stehen nicht in Hierarchie zueinander. Vielmehr handelt es sich um einen „zeitlich und sachlich gestaffelten Konkretisierungsmechanismus". ${ }^{72}$ Das Gerichtsurteil beendet einen Rechtserzeugungszyklus, der mit der legislativen Entscheidung als Formalisierung demokratischer Selbstbestimmung begann. ${ }^{73}$

Was aber, wenn ein Gericht ein Klimaziel, das vom demokratisch-legitimierten Gesetzgeber gesetzt worden ist, als ambitionslos verwirft, wie es im Falle von Urgenda der Fall war? Haben sich die Gerichte in diesem Fall nicht über die demokratisch-kollektive Selbstbestimmung des niederländischen Volkes hinweggesetzt? Hier stellt sich ganz grundsätzlich die Frage, in welchem Verhältnis Mehrheitsentscheidungen des Volkes zu den Grundrechten Einzelner stehen.

\section{Das Spannungsfeld zwischen Volkssouveränität und Menschenrechten}

Mit diesem Problem hat sich Habermas im Rahmen seiner Gleichursprünglichkeitstheorie beschäftigt. ${ }^{74}$ Demnach besteht ein Spannungsfeld zwischen einem „liberalen“ und „republikanischen“ Verständnis von Politik, zwischen der privaten und der öffentlichen Autonomie - oder auch: zwischen Menschenrechten und Volkssouveränität. Unter Privatautonomie wird nach liberaler Auffassung verstanden, dass jeder im Grunde frei ist, zu tun, was immer sie oder er will, solange dabei nicht in die Rechte anderer eingegriffen wird. Das Recht soll die freie Ausübung des individuellen Willens sichern. Rechte werden vor allem als Abwehrrechte verstanden, die das Individuum vor unzulässigen Eingriffen in sein Leben, seine Freiheit und sein Eigentum - durch tyrannische Mehrheitsentscheidungen - schützen sollen. Auf der anderen Seite räumt die republikanische Auffassung der öffentlichen Autonomie den höchsten Stellenwert ein. Unter öffentlicher Autonomie versteht Habermas die Möglichkeit, an Diskursen der Selbstregierung teilzunehmen, sich also an der kollektiven Selbstbestimmung einer politischen Gemeinschaft zu beteiligen, die nach

71 Nicht nur die Legislative und Judikative, sondern auch die Exekutive schafft Recht, siehe dazu Möllers, Gewalten (Fn. 68), S. $107 \mathrm{ff}$.

72 Möllers, Gewalten (Fn. 68), S. 117.

73 Möllers, Gewalten (Fn. 68), S. 116.

74 Vgl. dazu im Detail Habermas, Die Einbeziehung des Anderen. Studien zur politischen Theorie, 2. Aufl. 1997, S. 277-292 und Habermas, Faktizität (Fn. 35), insb. S. $151-165$. 
den Gesetzen lebt, die sie sich selbst gegeben hat. ${ }^{75}$ Diese Gesetze können wiederum zur Einschränkung der Privatautonomie Einzelner führen. Dieses Spannungsfeld wird auch in den Klimaklagen ersichtlich - der gerichtlich angestrebte Schutz der Grundrechte Einzelner vor den Auswirkungen des Klimawandels gegen das vom Gesetzgeber durch Mehrheitsentscheid gesetzte Klimaziel.

Was ist also wichtiger - die Menschenrechte oder die Volkssouveränität? Für Habermas sind die beiden Autonomien normativ gleichranging, keine genießt die Vorherrschaft über die andere. Vielmehr setzen private und öffentliche Autonomie, Menschenrechte und Volkssouveränität einander voraus. $^{76}$

Auf der einen Seite drückt sich im Willen des Gesetzgebers der deliberative Prozess der Entscheidungsfindung in der Bevölkerung aus. Die Bürger:innen haben die Gesetze selbst erschaffen und sind gleichzeitig jene, die ihnen unterworfen sind.

Auf der anderen Seite stehen die Menschenrechte, die zum einen die Privatautonomie schützen sollen. Sie sind darüber hinaus aber auch institutionelle Voraussetzung, dass jemand überhaupt am demokratischen Prozess teilnehmen kann. Das leuchtet unmittelbar ein für Kommunikationsgrundrechte, wie dem Recht auf Meinungsfreiheit, gilt aber auch für die anderen Menschenrechte: Wer gefoltert wird, wessen Leben bedroht wird, wer kein Zuhause hat, der hat andere Sorgen als wählen gehen oder sich in Diskussionen einzubringen. Menschenrechte ermöglichen und schützen die Teilnahme an diesen rationalen Diskursen. Sie stehen aber nicht einfach fest. Wie einfaches Recht auch, müssen sie von den Teilnehmer:innen rationaler Diskurse hinsichtlich ihrer Existenz, ihres Ausmaßes und ihrer Gestalt bestimmt werden. Hier schließt sich der Kreis und es wird ersichtlich, warum für Habermas Menschenrechte und Volkssouveränität nicht im Widerspruch zueinander stehen, sondern einander vielmehr bedingen. Beide sind gleichursprünglich für das Demokratieprinzip. ${ }^{77}$

Damit ist die Aufgabe des Gerichts definiert: Sie müssen sowohl die öffentliche als auch die individuelle Autonomie wahren. ${ }^{78}$ Das Volk will nicht von Richter:innen regiert werden, und das müssen die Gerichte akzeptieren. Wenn Richter:innen außertextliche Grundwerte „entdecken“, die ihre Rechtsprechung leiten, würden sie das demokratische Ideal des

75 Habermas, Einbeziehung (Fn. 74), S. 277-280.

76 Habermas, Faktizität (Fn. 35) S. 112.

77 Habermas, Faktizität (Fn. 35), S. 155.

78 Habermas, Faktizität (Fn. 35), S. 320. 
Verfassungsprojekts, wonach das Volk diese Werte selbst bestimmen will, verletzen. ${ }^{79}$ Somit ist die Justiz an das geltende Recht gebunden..$^{80}$

Das bedeutet aber nicht, dass Richter:innen das Gesetz bloß „vollstrecken“. Sie können bereits von der Gesellschaft vertretene Werte bekräftigen und neue Werte in die Rechtsordnung einführen, indem sie die Aufmerksamkeit auf Interessen lenken, die möglicherweise ungeschützt bleiben würden, weil sie entweder diffus und vage sind, wie z.B. Umweltrechte, oder die keine mächtige Lobby haben (z.B. arme und marginalisierte communities, zukünftige Generationen, aber auch Tiere und die Natur). Denn auch wenn das Volk nicht von Gerichten regiert werden möchte, so wenig steht es unter dem "government by the dead". ${ }^{81}$ Gerichte, insbesondere die Verfassungsgerichte, sollen auf die sozialen, technischen, ökonomischen und ökologischen Veränderungen reagieren können. Das BVerfG hat diese Lesart bestätigt:

„Hat der Gesetzgeber eine eindeutige Entscheidung getroffen, darf der Richter diese nicht aufgrund eigener rechtspolitischer Vorstellungen verändern und durch eine judikative Lösung ersetzen, die so im Parlament nicht erreichbar war. Die tatsächliche oder rechtliche Entwicklung kann jedoch eine bis dahin eindeutige und vollständige Regelung lückenhaft, ergänzungsbedürftig und zugleich ergänzungsfähig werden lassen. Die verfassungsrechtliche Zulässigkeit der Lückensuche und-schließung findet ibre Rechtfertigung unter anderem darin, daß Gesetze einem Alterungsprozeß unterworfen sind. Sie stehen in einem Umfeld sozialer Verhältnisse und gesellschaftspolitischer Anschauungen, mit deren Wandel sich auch der Norminhalt ändern kann. In dem Maße, in dem sich aufgrund solcher Wandlungen Regelungslücken bilden, verliert das Gesetz seine Fähigkeit, für alle Fälle, auf die seine Regelung abzielt, eine gerechte Lösung bereit zu halten. Die Gerichte sind daher befugt und verpflichtet zu prüfen, was unter den veränderten Umständen "Recht" i. S. des Art. 20 III GG ist. "82

79 Zurn, Law and Philosophy 2002, 467 (481).

80 Das ergibt sich für Habermas auch aus der grundsätzlichen Verschiedenheit zwischen den Begründungsdiskursen (der Legislative) und den Anwendungsdiskursen (der Judikative). Anders als in gerichtlichen Verfahren sind in den Begründungsdiskursen alle Beteiligte (denn theoretisch können alle Betroffenen an rationalen Diskursen teilnehmen). Zudem können die Gerichte die Repressionsmittel des Staatsapparats in Anspruch nehmen und verfügen damit über administrative Macht, vgl. Habermas, Faktizität (Fn. 35), S. 212 f.

81 Eisgruber, Constitutional Self-Government, 2001, S. 40.

82 BVerfG, Urt. v. 3.4.1990 - 1 BvR 1186/89, NJW 1990, 1593 f., ohne Nachweise. 
Eine besonders wichtige Rolle spielen hier die Menschenrechte. Sie können zu „Trümpfen" in der Hand von Einzelnen werden, die sie ausspielen können, um ihre Interessen gegen Nachteile, die das Resultat von politischen Mehrheitsentscheidungen sind, zu verteidigen. ${ }^{83}$ In solchen Fällen sollen Gerichte zugunsten des Einzelnen entscheiden dürfen. Wo Grundrechte verletzt werden, kann eine Mehrheitsentscheidung die private Autonomie gefährden - und damit letztlich die Demokratie im Allgemeinen. Zwar kann und wird nicht jedes Menschenrecht jede kollektive Entscheidung übertrumpfen, aber es setzt der Kosten-Nutzen-Analyse zugunsten von Kollektiventscheidungen doch eine Grenze. Dies folgt aus dem Prinzip der gleichen Achtung für jede Person. ${ }^{84}$

Insofern ist es nicht verwunderlich, dass die meisten Klimaklagen Menschenrechtsklagen sind. Burgers analysiert, dass das erstinstanzliche Urteil in der Urgenda-Trilogie, welches auf einer zivilrechtlichen Sorgfaltspflichten-Konstruktion basierte, sowohl in der niederländischen als auch internationalen Rechtswissenschaft teils stark kritisiert wurde, wohingegen die Urteile des Berufungsgerichts und des Hohen Rates viel positiver aufgenommen worden seien. ${ }^{85}$ Das Berufungsgericht hat die Dynamik zwischen Volkssouveränität und Menschenrechten in Urgenda II explizit angesprochen:

"The State argues that for this reason the system of the separation of powers should not be interfered with, because it is not up to the courts but to the democratically legitimised government as the appropriate body to make the attendant policy choices. This argument is rejected in this case, also because the State violates human rights, which calls for the provision of measures, while at the same time the order to reduce emissions gives the State sufficient room to decide how it can comply with the order. "86

\section{Abschließende Bemerkungen}

Die Verfassung ist ein unabgeschlossenes Projekt. Sie muss immer neu und besser - interpretiert werden. ${ }^{87}$ Das betrifft auch die Einbeziehung ökologischer Entwicklungen, die zum Zeitpunkt ihres Inkrafttretens noch

83 Dworkin, Taking Rights Seriously, 1977, S. XI.

84 Habermas, Faktizität (Fn. 35), S. 250.

85 Burgers (Fn. 26), 64-66.

86 Urgenda II (Fn. 4), Rn. 67.

87 Habermas, Faktizität (Fn. 35), S. 464. 
nicht absehbar waren. Bei dieser Aktualisierung hilft den Gerichten auch ein Blick auf die europa- und völkerrechtlichen Verpflichtungen sowie wissenschaftlichen Entwicklungen.

So lag das (damalige) EU-Klimaziel bei $20 \%$ und damit höher als das angestrebte Klimaziel der Niederlande. Die Gerichte haben diese europarechtliche Verpflichtung bei ihrer Argumentation mitzugrunde gelegt. ${ }^{88}$ Viele Staaten sind zudem völkerrechtlichen Verträgen wie der UN-Klimarahmenkonvention (UNFCCC) oder dem Pariser Übereinkommen beigetreten. Diese Verträge spielen oft eine wichtige Rolle, denn in den meisten erfolgreichen Klimaklagen haben Gerichte Menschen- und Grundrechte im Lichte umweltvölkerrechtlicher Verpflichtungen interpretiert.

Auch einseitige Erklärungen können ein Gericht bewegen, den Gesetzgeber zu mehr Klimaschutz zu verurteilen. So hatte Kolumbien im Vorfeld des Paris Agreement von 2015 versprochen, die Abholzung des Amazonas zu reduzieren. Nachdem dies in den Folgejahre nicht geschehen ist, hat der kolumbianische Corte Suprema de Justicia die Regierung 2018 in einem aufsehenerregenden Urteil, mit dem er zudem den Amazonas zu einem Subjekt eigener Rechte machte, verurteilt, eine Zero Deforestation Policy auszuarbeiten und umzusetzen. ${ }^{89}$ In Pakistan verurteile ein Gericht die Regierung 2015, das Land „climate-proof“ zu machen. Dabei ging es dem Gericht vor allem darum, dass der Staat bereits existierendes nationales Klimarecht endlich umsetzt.

Es wird hier deutlich, dass Gerichte Klimaverpflichtungen nicht aus der Luft greifen, sondern auf nationale und internationale Verpflichtungen verweisen, die die Staaten freiwillig eingegangen sind. Die Verfügbarkeit von immer mehr klimawissenschaftlichen Erkenntnissen, insbesondere den Berichte des IPCC, helfen den Gerichten wiederum, die Gefahren des Klimawandels einzuordnen und den Schutzbereich von Menschen- und Grundrechten bzw. dessen Verletzung zu bestimmen. Die Art und Weise, wie die Regierungen diese Menschenrechtsverletzungen beseitigen, wurde ihnen aber ins Ermessen gestellt. All diese Gesichtspunkte entschärfen den Vorwurf, dass Gerichte Klimarecht gegen den Willen des Gesetzgebers schaffen würden.

Daneben ist die grundsätzliche Diskussion, ob ein Gericht einem bestimmten Partikularinteresse tatsächlich den Vorrang vor anderen geben darf, noch nicht beendet. Allerdings stellt sich hier wiederum die Frage,

88 Vgl. Urgenda I (Fn. 3), Rn. 4.25 f.

89 Future Generations, Rn. 11.3. 
ob der Klimaschutz wirklich ein weiteres Partikularinteresse, und damit vergleichbar z.B. mit wirtschaftlichen Interessen, ist.

UN-Sonderberichterstatter Alston weist darauf hin, dass Staaten konsequent schlechte wirtschaftliche Argumente nutzen, um das Nichtstun gegen den Klimawandel zu rechtfertigen. ${ }^{90}$ Dabei hat bereits 2006 der Ökonom Stern vorgerechnet, dass ein „Weiter so“ die britische Wirtschaft jedes Jahr mindestens 5\% (und möglicherweise bis zu 20\%) des Bruttoinlandprodukts (BIP) kosten würde. ${ }^{91}$ Dem IPCC zufolge würde die Welt bei einer Erwärmung um $2^{\circ} \mathrm{C}$ sozioökonomische Verluste in Höhe von 13\% des globalen BIP und Schäden in Höhe von 69 Billionen Dollar erleiden. ${ }^{92}$ Insgesamt hängen 1.2 Milliarden Arbeitsplätze (40\% der weltweiten Beschäftigung) von einer nachhaltigen und gesunden Umwelt ab. Der Hitzestress sorgt selbst im günstigsten Fall $\left(1,5^{\circ} \mathrm{C}\right.$-Szenario) für eine Reduzierung der globalen Arbeitszeit um 2\% bis 2030. ${ }^{93}$

Der Klimawandel hat das Potential, nicht nur die Wirtschaft nachhaltig zu schaden, sondern eine „Klima-Apartheid“ auszulösen und den Rechtsstaat und die Menschenrechte, ${ }^{94}$ schlussendlich sogar die menschliche Zivilisation auszulöschen. ${ }^{95}$ Die Bewahrung unserer natürlichen Grundlagen, ohne die ein menschenwürdiges Leben überhaupt nicht möglich ist, steht somit im Interesse aller. Anstatt also weiterhin künstlich Klimaschutz gegen Wirtschaftsinteressen abzuwägen, wäre vielmehr erforderlich, dass die Voraussetzungen für eine echte grüne Wende geschaffen werden. Andernfalls hat Sachs, ein führender US-amerikanischer Ökonom, einen Vorschlag an die Bürger:innen, wie man mehr für den Klimaschutz tun könne: sie sollen die Gerichte mit Klagen „fluten“, um das Recht auf eine gesunde Umwelt durchzusetzen. ${ }^{96}$

90 Special Rapport “Climate Change and Poverty" (A/HRC/41/39), 2019, Rn. 38.

91 Stern, The Stern Review on Economics of Climate Change: Summary of Conclusions, 2006, S. 1.

92 IPCC, Sonderbericht Global Warming of $1.5^{\circ} \mathrm{C}, 2018$, S. 264.

93 ILO, Greening with Jobs, 2018, S. 2, 7, 27.

94 Special Report (Fn. 91), Rn. 50, 65.

95 Vgl. NSW Land and Environment Court, Walker v. Minister for Planning (NSWLEC 741/157 LGERA 124) Rn. 161: "Climate change presents a risk to the survival of the human race and other species. Consequently, it is a deadly serious issue"; Abweichende Meinung von Richter Staton im Fall Juliana (Fn. 42), spricht von der "Nation's willful destruction" (S. $33 \mathrm{ff}$ ).

96 Sachs, A Proposal for Climate Justice, Vortrag v. 10.10.2017 an der London School for Economics. 